\title{
An Evaluation of Be Strong: A Nurse-Led Caregiver- Assisted Exercise Program to Reduce Fall-Risk in Older Adults
}

\author{
Cindy Gross* \\ DNP RN PHN CEN NEA-BC, Gonzaga University, Lake Forest CA, USA
}

Submission: April 22, 2018; Published: May 02, 2018

*Corresponding author: Cindy Gross DNP RN PHN CEN NEA-BC, Gonzaga University, 502 E. Boone Ave, Spokane, WA 99258, Bright Star Care of Lake Forest, 25951 El Toro Road, Lake Forest CA 92630, USA, Tel: (949) 412-4018; Email: Tlchealtheduc@aol.com

\begin{abstract}
One in three adults over age 65, fall each year sustaining an injury CDC [1]. Falls can be reduced with regular exercise; yet, confidence in balance and fear of falling can psychologically limit older adults from participating in mobility. This nurse-led caregiver-assisted exercise pilot project evaluates fall-risk reduction, confidence in balance and fear of falling. Nine participants engaged in 20 minutes of daily tailored caregiverassisted exercise over a 20-week period. Data were analyzed using descriptive and inferential statistics. The Berg Balance Scale and Timed Up and Go test showed statistically significant differences in fall-risk scores. The Activities-Specific Balance Confidence Scale, Fall Efficacy Scale and Fall Efficacy International Scale showed no statistically significant difference in confidence and fear of falling, however clients continued the exercise program and data supported frail elderly concerns on fear of falling. Participants, agency staff and consultants evaluative survey showed positive feedback of this exercise experience.
\end{abstract}

Keywords: Older Adults; Fall-Risk; Exercise; Caregiver

Abbreviations: FESI: Fall Efficacy Scale International; CDC: Disease Control and Prevention; BOD: Board of Directors; PAC: Professional Advisory Committee; BBS: Berg Balance Scale; TUG: Timed Up and Go; MMSE: Mini Mental State Exam; ABC: Activities-Specific Balance of Confidence

\section{Introduction}

Older adults, age 65 and over, comprise the fastest growing segment of the American population (Centers for Disease Control and Prevention CDC [2]. In the U.S., there are currently 43.3 million older adults. This number is estimated to double by 2050 U.S. Census [3]. The U.S Department of Health and Human Services (2014) aims to promote health, well-being, a sense of belonging and independence for this aging population. Many challenges facing older adults are acute care, chronic disease management, nursing home admissions and loss of independence. Contributing to these issues is a growing concern about falls, functional ability and aging safely Roper [4]. The CDC [1] states that one in three older adults fall each year resulting in 2.4 million injuries. Of these individuals, 722,000 are hospitalized and 22,500 die. The annual healthcare cost associated with falls is $\$ 30$ billion. It is estimated that with the expansion of this population, the cost of care will reach $\$ 54$ billion by 2030 CDC [2]. Falls are a major threat to the physical health, psychological independence, and quality of life of older adults. Falls are preventable CDC [5]. A meta-analysis of older adults' mobility, activity and fall-efficacy by Schepen et al. [6] found that the fear of falling psychologically limited activity in older adults and contributed to increase fall- risk. Faes et al. [7] interviewed a group of frail elderly adults who had sustained a fall. A constant fear of falling, the fear of unknown, consequences from falling, the loss of physical capabilities, and the loss of independence were the most common themes. These fears negatively affect quality of life Huang et al. [8]. Makizako et al. [9] showed that older adults are more vulnerable to muscle weakness, gait impairment, poor balance and fall-risk when they are afflicted by limited mobility and a fear falling.

A key variable to reduce falls with the aging population is exercise CDC [2]; Gillespie et al. [10]. Exercise and mobility are essential for physical, mental health, and overall wellness. In systematic reviews, meta-analyses and randomized controlled trials, physical activity improved physical function, muscle strength, gait, and balance. This key item is needed to prevent falls in older adults CDC [1]; Huang et al. [8]; Baruth et al. [11]. Fall reduction rates of $17 \%$ to $42 \%$ were shown in studies with older adult exercise. Elderly involved in challenging balance exercises showed the greatest improvement Day et al. [12]; Sherrington [13]. In collaborative studies of 13,264 subjects, fall- reduction rates of $32 \%$ were shown with home-based exercise programs. These older adults engaged in exercise in the privacy of their 
home Gillespie et al. [10]. Robertson et al. [14] showed a 33\% fall reduction in home-based tailored exercise programs. Under the watchful eye of licensed healthcare members, home-based resistance training exercises showed a $33 \%$ to $67 \%$ improvement in older adult functional strength Thiebaud et al. [15].

\section{Pilot Project}

As a DNP project, Be Strong was created and developed by an interdisciplinary team as a nurse-led, caregiver assisted exercise program for fall-risk older adults. The program focused on teaching older adults at-risk for falls, innovative ways to successfully exercise at home. The team consisted of three physical therapists, one fitness trainer and one doctoral student registered nurse. Collaboratively, this team created and developed a library of 10-12-minute videos consisting of sitting, laying and standing exercises. The aim of these videos was to provide older adults and caregivers with an ability to visually model tailored exercises of short duration. The goal of modeling innovative exercise behaviors served to reduce fall-risk through improved muscle strength and balance.

Resnick's Self-Efficacy Nursing Theory forms the foundation for the conceptual framework of Be Strong's nurse-led caregiver assisted-exercise program. It is designed for older individuals who have an attitude, belief, and a willingness to engage in exercise, but who may be reluctant due to fear of falling. Clients and guardians presented with education and evidence supporting fall risk with aging and inactivity were provided a choice to engage in Be Strong exercise program with their caregiver's assistance. The emotional legitimacy of the fear of falling among fall-risk older adults, and relationship of positive encouragement, guidance and safety by caregiver support, offer older frail clients a choice to model and practice healthy exercise behaviors at home as a fall-reduction strategy (CDC, 2015) Resnick et al. [16].

This Be Strong project was proposed and offered to clients of Bright Star Care of Lake Forest who were of age 65 and older and at-risk for falls. Bright Star Care is a California licensed and Joint Commission accredited home-health agency providing skilled licensed nursing services as well as non-skilled caregiver services for clients in Southern California. The Board of Directors (BOD), Professional Advisory Committee (PAC) approved this nurse-led caregiver assisted exercise pilot project. Participants of age 65 and over, at- risk for falls and with caregiver support, were screened from Bright Star Care's active client list. Those at-risk clients and guardians of at-risk clients were contacted and informed of the project by the doctoral student to determine if there was an interest and willingness to engage in caregiver assisted exercise. In addition to Bright Star Care's clients, additional elderly were added to the program through word of mouth, primary care referrals and church organizations. Clients were fully informed of the exercise project and consent was obtained. Guardian consent was sought for individuals with low scores in the Mini Mental Health Exam and Katz Independence of Function Scale. All participants were subsequently admitted as clients to Bright Star Care. All client contact and client / caregiver education was voluntarily provided at no cost or compensation by the doctoral student for the entirety of the project. The Be Strong program evaluation was conducted as a Doctorate of Nursing Practice (DNP) scholarly project and was approved by Gonzaga University's Institutional Review Board. The setting for conducting Be Strong exercises was at the client's home with caregiver support.

Clients and caregivers received home visits from the nurse to evaluate the location in the home where the exercises would be conducted, the ability for the videos to be viewed and the one-toone training for clients and caregivers. Data collected by the nurse on the client fall risk, confidence and fear of falling aided in the selection of two 10-12 minute exercises from the Be Strong library of videos. These exercises were instructed to be done separated in time or together for a total of 20 minutes daily. Caregiver training emphasized client assistance in modeling the exercises performed by the fitness trainer on the video. Additionally caregiver training centered on providing positive reinforcement, encouragement and praise to the client for healthy exercise behavior to aid in building confidence and lessen the fear of falling. Clients and caregivers were provided telephone contact with the nurse and communicated every week to discuss progress or lack of progress in the assigned exercises. This time period determined if clients were able to master these two assigned 10 to 12-minute exercise sessions or if modifications were needed to the exercises. Clients who did not want to engage in exercise could refrain from exercise on any day or withdraw from the program at any time.

\section{Methods/Measures}

Demographic data were collected at the start of the project and included age, gender, medical history, the Mini-Mental State Exam and the Katz Index of Independence of Activities in Daily Living Index. The Mini- Mental State Exam (MMSE) evaluates cognitive ability in orientation, memory, recall, attention, naming, reading, writing, copying figures and following simple instructions. The maximum score is 30 and a score of 23 or lower indicates cognitive impairment. The Katz Index of Independence evaluates functional independence ( 1 point) or dependence ( 0 points) on activities of bathing, dressing, toileting, transferring, continence and feeding. A score of 6 indicates independent functioning and 0 indicates dependent functioning. Enrolled clients were instructed to exercise daily with their caregiver's assistance using two 1012- minute video-assisted exercises over a period of 20 weeks. Fall-risk data were measured at four week intervals evaluated and aided in changing the clients video exercise plan. Each exercise video was tailored and selected specifically for each client based on their cognitive, functional and measures. Confidence in balance and fear of falling data were collected less frequently at eight week intervals to allow the client to focus on muscle strengthening exercises, feel secure with their caregiver support and reduce the focus of falling with exercise. These measures were collected over the 20-weeks of exercise. Modifications in exercise were made for cognitively challenged clients, having the caregiver follow 
the video to assist client modeling. Sitting and laying exercises were assigned for cognizant clients on days when the caregiver was not available to assist them with exercise. On caregiver assisted days, these clients were assigned and engaged in more challenging exercises. This strategy provided an affordable and safe alternative to promote healthy daily exercise behaviors. Both clients and caregivers received one-to-one education by the nurse with every change in exercise. The focus was on review of the new exercises, monitor client endurance, fatigue, safety, guarding and encouragement (Figure 1). At the completion of the exercise project, measures of the BBS, TUG, ABC, FES and FESI were analyzed for differences in fall-risk, confidence in balance and fear of falling.

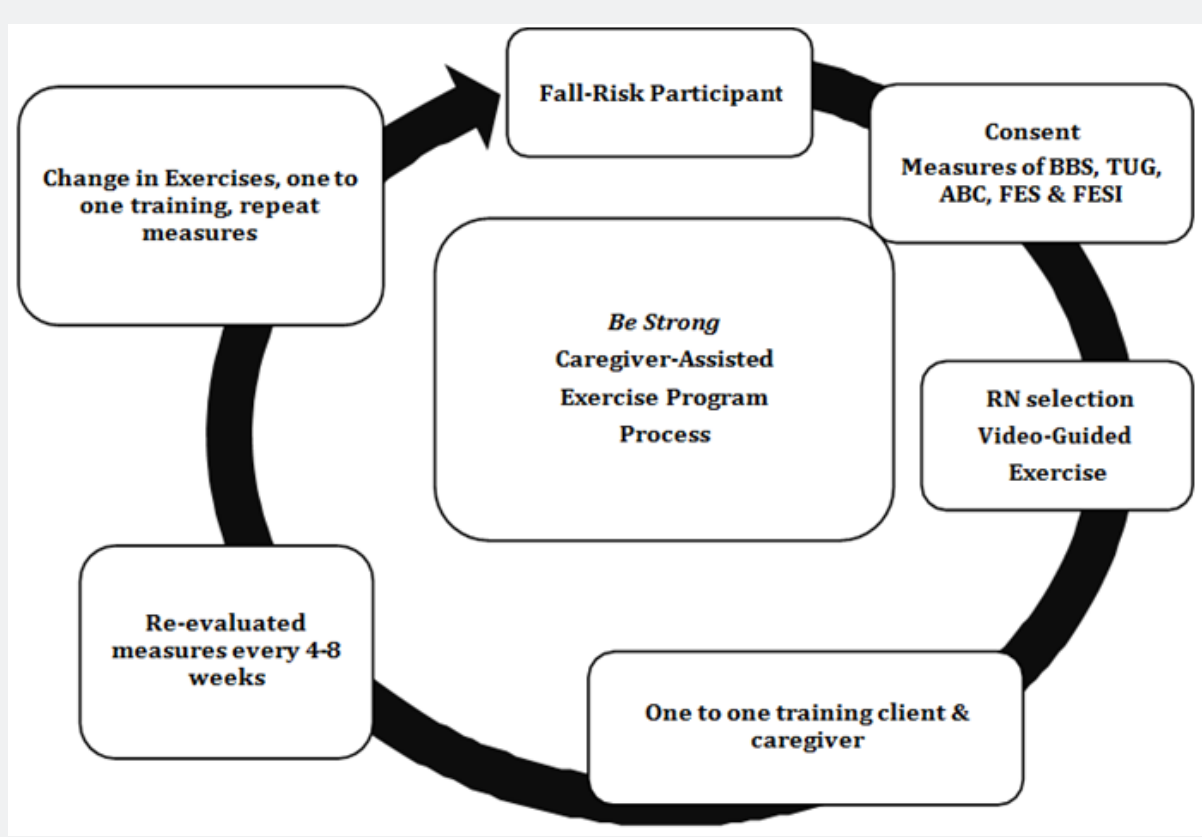

Figure 1: Be Strong Process for Client/Caregiver Engagement in Assisted Exercise.

The Berg Balance Scale (BBS) and Timed Up and Go (TUG) tests were used to screen fall risk and eligibility of consenting participants. The Berg Balance Scale (BBS), a valid and reliable tool measured 14 balance tasks rated on a scale from 0 to 4 . The sum of all 14 measures ranged from 0 to 56 points. The lower the score, the greater the client's fall-risk. The higher the score, the better performance in the balance tests. The Timed Up and Go (TUG) test, another valid and reliable tool, tool, measured client mobility and fall risk. The task required the client to rise from a standard chair, walk 15 feet, then return to a sitting position in the same chair. This test is measured in seconds of time to accomplish a task. Higher scores (longer time) indicate mobility limitations and higher fall-risk.

The Activities-Specific Balance of Confidence (ABC) selfassessment scale measures client confidence in balance performing 16 functional daily activities. Clients rated confidence levels with performing tasks on an ordinal scale from $0 \%$ of the time to $100 \%$ without losing balance. Scores of $80 \%$ or greater indicate a high level of physical function, $50 \%-80 \%$, a moderate level and less than $50 \%$ a low level of physical function. A low score indicates an increased fear of falling. The Fall Efficacy Scale (FES) asks clients/caregivers to self- rate the ability to perform 10 daily activities without falling on a 1 to 10 scale. Ratings of very confident are $=1$, not confident at all $=10$. Scores computed as the sum greater than 70 indicate a high fear of falling. The Fall Efficacy Scale International (FESI) is a self-rated tool to determine the degree of concern about falling on a Likert scale based on performing 12 daily activities. A scale of 1 (not at all concerned) to 4 (very concerned) produce collective scores indicative of low concern ranging 12-19, a moderate concern 20-27 and a high concern 28-48 of falling. Survey questionnaires were provided to all direct and indirect supporting members of this project at its completion. Members included clients, family/caregivers, agency staff and consultants. Clients and caregivers received a 15-item survey with a five point Likert scale. Items on the survey asked about the enrollment process, the explanation of the program by the nurse and the consent process, as well as the quality of the videos, the selection of the videos and the effects of the exercise on muscle strength, balance and confidence. Agency staff received a six-item survey on a 5-point Likert scale evaluating the impact of client enrollment, hiring staff and communication with the team. The consultants received a six-item survey on a 5-point Likert scale evaluating their roles, communication, video production and the relevance of this exercise project for older adults. All surveys contained a comment section for free scribed responses.

\section{Data Analysis and Results}

Data were analyzed for nine inclusive exercise clients on demographic age, gender, medical history, cognitive and 
functional status, fall-risk, confidence in balance and fear of falling. Comparisons of pre- exercise and post-exercise measures were used utilizing t-testing to determine statistical differences.

Feedback from the evaluative survey was analyzed for qualitative and quantitative results.

\section{Demographic}

Eleven clients were screened for inclusion to this project. Reasons for exclusion were those less than 65 years of age, no fall risk and those who were not interested in this exercise program. One client declined participation after 3 weeks due to disinterest and a second client expired. Of the nine consenting participants to the Be Strong nurse-led caregiver assisted exercise program, three $(n=3)$ were males and six $(n=6)$ were females. The age range was 81 to 91 with a mean of 86.4. This population resulted in the over 80 elderly adults at-risk for falls. The medical/surgical history on the clients indicated two clients $(\mathrm{n}=2)$ with a history of cardio/ pulmonary disease and three clients $(n=3)$ with only pulmonary disease, both groups of which were treated with medication and medical management. Three clients $(n=3)$ had neurological histories of transient ischemic attacks, previous subdural hematoma, and cervical stabilization, while three $(n=3)$ clients had history of prior orthopedic surgeries. Physician clearances were obtained for clients to participate in this Be Strong exercise program. The Mini-Mental State Exam (MMSE) indicated 56\% $(n=5)$ were cognitively impaired and $44 \%(n=4)$ were without cognitive impairment at the start and end of the program. The Katz Index of Independence showed 33\% ( $n=3)$ of participants functioned at a high level, 22\% (n=2) moderate and $44 \%(n=4)$ at a low level of independent function with a mean score of 3.1 at the start of the program. Two clients were hospitalized during weeks 16 to 20, but remained in the project. Due to client hospitalization, the Katz Index of Independence data resulted with 33\% (n=3) of participants functioned at a high level, $11 \%(n=1)$ moderate and $55 \%(n=5)$ at a low level of independent function with a mean score of 2.6 at the conclusion of the program.

\section{Fall Risk}

Of the nine participants, at 20 weeks of exercise, the overall increase in BBS points ranged from 3 to14. At week 20, one hospitalized client lost 5-points and the second client lost 19-points from their BBS pre- score. At week 16, prior to hospitalization, the first hospitalized client showed a 3-point gain and the second client showed an 11-point gain on BBS. The maximum gain in BBS points for these two clients were 5 points for the first client and 11 points for the second client. With regards to $\mathrm{BBS}$, the post-score $(\mathrm{M}=32.67, \mathrm{SD}=16.12)$ was higher than the pre-score $(M=26, S D=9.87)$, indicating a difference marginally significant, $\mathrm{t}(8)=1.75, \mathrm{p}=0.0591$ (one-tailed). Analyzing 7 clients at week 20, excluding the two hospitalized clients, the BBS post-score $(M=38.71, S D=2.94)$ was higher than the prescore $(M=26.71, S D=11.25)$ indicating a statistically significant positive difference, $t(6)=10.78, p=0.0000$ (one-tailed). Analyzing data for seven clients at week 20 and two hospitalized clients at week 16 , BBS post-score $(M=36.88, S D=9.87)$ was higher than the pre-score $(M=26, S D=9.87)$, indicating a statistically significant positive difference, $t(8)=8.33, p=0.0000$ (one-tailed). With regards to TUG time for all 9 clients, TUG post-score $(\mathrm{M}=26.28$, $\mathrm{SD}=19.09$ ) was lower than the TUG pre-score ( $M=35.76$, SD $=20.44$ ), indicating a reduction in time; however, the difference was not statistically significant, $\mathrm{t}(8)=1.14, \mathrm{p}=0.1445$ (one-tailed). TUG time for 7 clients at week 20, excluding the two hospitalized clients, TUG post-score ( $\mathrm{M}=18.28, \mathrm{SD}=8.17)$ was lower than the TUG pre-score $(\mathrm{M}=37.56, \mathrm{SD}=23.02)$ indicating a reduction in time; a statistically significant positive difference, $t(6)=2.98$, p $=0.0249$ (one-tailed). TUG time for 9 clients, 7 at week 20 and 2 hospitalized clients at week 16 , TUG post-score (M $=20.98$, SD =9.15) was lower than the TUG pre-score ( $M=35.75, \mathrm{SD}=20.44$ ) indicating a reduction in time; a statistically significant positive difference, $\mathrm{t}(8)=2.46, \mathrm{p}=0.01975$ (one-tailed).

\section{Confidence in Balance}

At the start of the program, $78 \%(n=7)$ of the participant scores indicated a low level of function in balance on the $A B C$ scale. At 20 weeks of exercise, $44 \%(n=4)$ scored a low level of function, an improvement of 34\%. The ABC post-scores for all 9 clients $(M=43, S D=26.78)$ was higher than the $A B C$ pre-score ( $M$ $=34, \mathrm{SD}=22.59$ ), indicating a higher level of function; however, the difference was marginally statistically significant, $t(8)=1.74$, $\mathrm{p}=0.06015$ (one-tailed).

\section{Fear of Falling}

Participant scores on the FES scale revealed equal pre-and post-exercise scores of $22 \%(n=2)$ with a fear of falling and $78 \%$ $(\mathrm{n}=7)$ with no fear of falling. FES post-scores for all 9 clients $(\mathrm{M}=42.33, \mathrm{SD}=32.36)$ was lower than the FES pre-score $(\mathrm{M}=$ $47, \mathrm{SD}=27.78$ ), indicating a lower fear of fallings; however, the difference showed no statistically significant difference, $t(8)=$ $0.53, \mathrm{p}=0.30645$ (one-tailed). The FESI scale showed 33\% ( $\mathrm{n}=3$ ) with a high fear of falling (FOF), 44\% (n=4) low FOF, $11 \%(n=1)$ no change in FOF and $11 \%(n=1)$ incomplete data. FESI pre-score (M $=31.13, \mathrm{SD}=11.91)$ was lower than the FESI pre-score $(\mathrm{M}=31.38$ $\mathrm{SD}=9.26)$, indicating no statistically significant difference, $\mathrm{t}(7)=$ $0.08, \mathrm{p}=0.46825$ (one- tailed).

\section{Program Evaluation}

Clients and caregivers received the same 15-item survey questionnaire. The clients without cognitive impairment were four $(n=4)$ and caregivers/family members were eight $(n=8)$. Overall responses to enrollment in the Be Strong program, the explanation of project and the consenting process were rated as very easy to easy. The frequency of nurse assessments and home visits were rated by both groups as very helpful. Two clients experienced inconsistencies with committed caregiver assistance. Exercises were modified for these clients with safe sitting or lying down with exercises on days without assistance. These clients chose not to response to the questions related to caregiver 
assistance. One client found that having a caregiver assist with exercise and the presence of the assisting caregiver as very helpful while the second client rated this as helpful. The ongoing followup nurse home visits were rated as very helpful and helpful by both clients and caregivers. The selection of and the quality of the exercise videos were rated as very helpful and helpful. Three of four clients $75 \%(n=3)$ and $88 \%(n=7)$ of caregivers found the exercise program had a lot of effect on muscle strength. $75 \%$ $(n=3)$ of clients and $100 \%(n=8)$ of caregivers found the exercise program had a lot of effect on balance. $75 \%(n=3)$ of clients and
$100 \%(n=8)$ of caregivers found the exercise program affected confidence a lot (Figure 2). A survey question asked clients how often they would exercise after completing this project. Four clients without cognitive impairment responded to this question $25 \%(n=1)$ indicated that they would exercise daily, 25\% (n=1) 5 times per week and $50 \%(n=2)$ three times per week. Seven caregivers responded to the same question for clients with cognitive impairment and 71\% ( $n=5)$ indicated the client would exercise with them daily, $14 \%(n=1) 5$ times per week and $14 \%$ $(n=1) 3$ times per week (Figure 3).

\section{What effect did Be Strong have on my... muscle strength...confidence...balance}

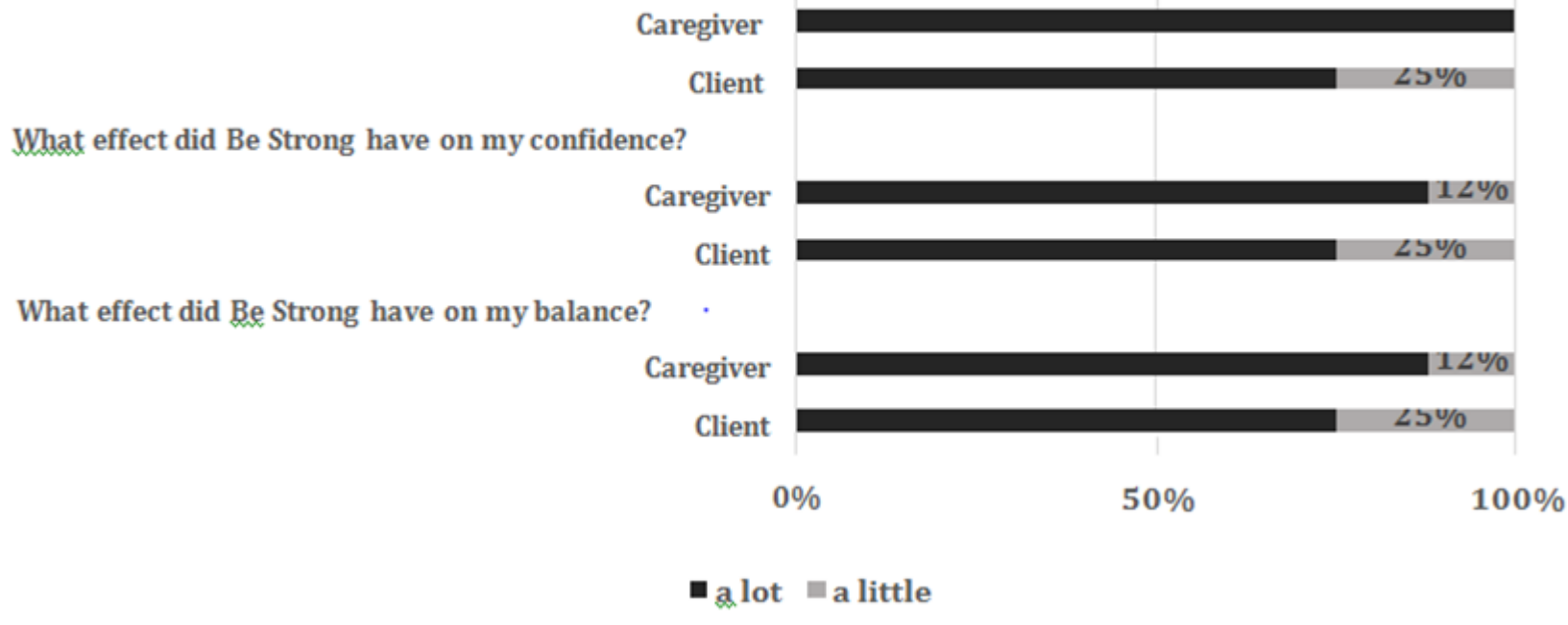

Figure 2: Perceived Effects of Be Strong on Muscle Strength, Confidence and Balance.

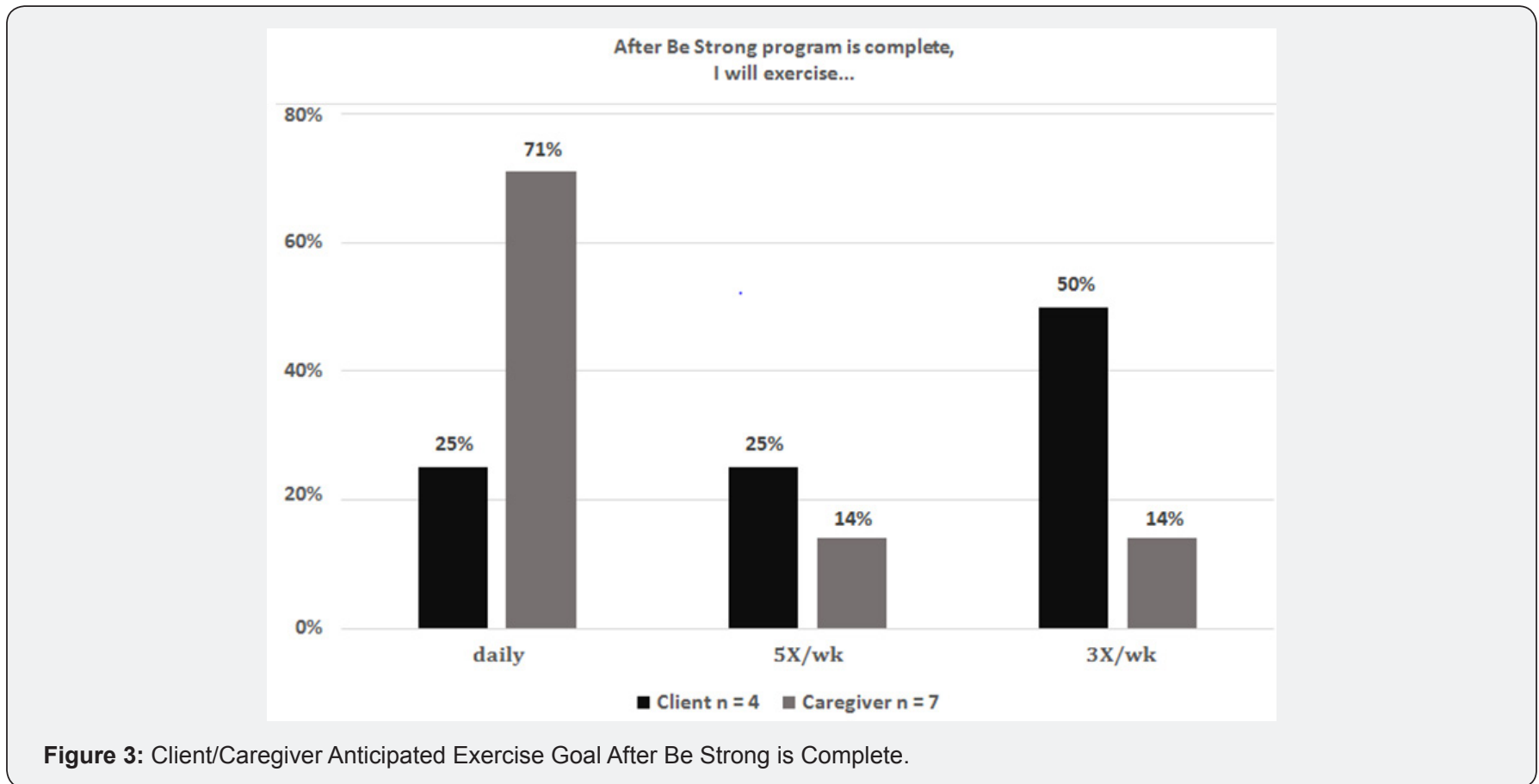


A 6-item survey questionnaire was provided to three $(n=3)$ agency members, the president/CEO, care coordinator and staffing coordinator. Their perception of managing their current client's enrollment to the Be Strong exercise program was rated as either very easy $33 \%(n=1)$, easy $33 \%(n=1)$ or okay $33 \%(n=1)$. Their management of new client's enrolled to the program was rated as very easy $67 \%(n=2)$ and okay $33 \%(n=1)$. One comment indicated that it "did not adversely impact our operations." The hiring/ staffing of caregivers for the exercise program was rated very easy $67 \%(n=2)$ and easy $33 \%(n=1)$. The frequency of staff meetings for updates on the exercise program was excellent $33 \%(n=1)$ and good $67 \%(n=2)$. The ongoing communication with the agency staff and the ease of implementing the exercise program was rated as excellent $67 \%(n=2)$ and good $33 \%(n=1)$. It was noted that the process "was seamless for our operations." Four consultants were provided with an evaluation and rated their consultant role as excellent $50 \%(n=2)$ and $50 \%(n=2)$ rated it as good. The frequency of meetings was rated $25 \%(n=1)$ excellent, $50 \%(n=2)$ good and $25 \%(n=1)$ fair. The quality of the communication as an interdisciplinary team, $50 \%(n=2)$ rated excellent, 50\% $(n=2)$ rated good. The following three questions: The quality of the videos produced, the usefulness/relevance of the exercise videos and the effectiveness of the videos for older adult exercise were $100 \%(n=4)$ rated as excellent. One consultant shared: "The Be Strong video library is very well done. I am impressed with the content, the diversity of exercises, and its ability for application to an adult population at risk for falls. Having a number of videos allows (permits) multiple starting points and allows participants to progress to more challenging activities. The pace of the video seemed very appropriate for older adult learners..."

\section{Discussion}

Older adults are the fastest growing segment of the American population. Health, independence, and functional living can be affected by a fall event impacting the quality of life. Be Strong's evidenced-based nurse-led program managed, measured and tailored caregiver-assisted exercises for successful fall-risk reduction. This DNP scholarly project offered 20-24 minutes a day of a nurse-led caregiver-guided exercise program aimed at muscle strengthening and balance for frail fall-risk elderly clients. Nursing assessment of fall-risk elderly with a plan for appropriate safe caregiver assisted exercise intervention, promotes health, strength, balance and aims to maintain functional abilities for safe living. On days when cognitive clients did not have caregiver assistance, select sitting and laying exercises encouraged consistence in daily exercise. An agreement with an academic nursing program permitted RN to BSN nursing students to provide community service to assist frail elderly at home with exercise. A partnership with nursing programs serve as an outreach of community health service. In the privacy of the clients' home, regularity with assisted exercise improved BBS and TUG scores. Be Strong participant's functional status remained unchanged with the exception of the two client hospitalizations resulting in one client reducing functional status. As some Be Strong participants improved strength, they requested more challenging exercises, which supported one consultant comment stating "...They (clients) want to be challenged at any level and I know they can be successful..." The results of this pilot project at should be cautiously considered as it was implemented on a voluntary basis as a scholarly doctoral project by the doctoral student at one organization with a small number of clients. Nonetheless, the results of this pilot exercise project reflects the literature review that regular physical activity improves strength and balance as a fall reduction strategy concluded by CDC [1]; Huang et al.[8]; Baruth et al. [11]; Gillespie et al. [10]; Day et al. [12]; Sherrington et al. [13]; Robertson et al. [14]; Thiebaud et al. [15]. The psychological impact of fear of falling with frail elderly was an important consideration expressed by Faes et al. [7]. The use of reliable ABC, FES and FESI scales resulted in no statistically significant difference in the fear of falling verifying the serious impact of falling on frail elderly. Although the survey questionnaire indicated $75 \%$ of clients $(n=3)$ and $100 \%$ of caregivers $(n=8)$ positively responded that Be Strong affected their confidence a lot, the respondent survey data has limitations, perhaps due to evaluating 15 items in sequence. It is, though, a recommendation to use of a valid instrument to secure accurate data on client confidence and fear of falling $[17,18]$.

Although Be Strong nurse-led caregiver-assisted exercise project was implemented at Bright Star Care of Lake Forest, it was done voluntarily as a DNP scholarly project by a doctoral student RN. Furthering of this project includes training the agency RNs and providing caregiver certification to standardize the program for consistent screening of at-risk fall clients, the proper use of measurement tools, and the appropriate selection of videos for replicative results. Consultation from the project team will evaluate the continued use of multiple measurement tools for fall risk and fear of falling. Future sharing of this program with corporate Bright Star and other home health agencies expansion and evaluation this program with greater client volume over a greater time frame. The cost for implementing this model in home health includes RN training, home visits and caregiver training. Agencies ability to bill for such service will be at client expense until it is recognized as a nursing billable service. Family based caregivers can reduce cost by certified training to assist with exercise. Supportive data on this strategy of fall reduction encourages state and federal proposals to examine Be Strong nurse-led home-based exercise as a billable service. Shifting dollars spent on fall injuries to assisted exercise seems an encouraging option to improve the quality of life for elderly. Be Strong nurse-led caregiver-assisted exercise program contributes to advancing nursing practice by promoting healthy exercise behaviors with frail elderly offering, safe physical activity at home with a trained assisting caregiver. Optimistic success in experiencing strength and improved balance, encourages Be Strong future as a fall-reduction strategy $[19,20]$.

\section{Conclusion}

Be Strong nurse-led exercise project taught older adult's at-risk for falls, innovative ways to successfully exercise with 
caregiver assistance and consequently reduce fall-risk. The use of caregiver assistance and video-based exercises of short duration allowed older frail adults to model tailored exercises with positive gains in strength and balance. The results of the BBS and TUG showed a statistically significant positive difference in reducing fall-risk scores after 20 weeks although no statistically significant difference resulted for confidence in balance and fear of falling. The continuation of this program will serve as a contribution to improving older adult healthy living and quality of life.

\section{Acknowledgement}

Special thanks and acknowledgement to my Family, Gonzaga University Faculty, Bright Star Care of Lake Forest California, and consultants Michael Bennett, Thomas Dillon, Nancy Evanston and Ronald Williams. With a great deal of respect and gratitude, I express a heartfelt thanks and appreciation.

\section{References}

1. Centers for Disease Control and Prevention (2013) Older adult falls data and statistics.

2. Centers for Disease Control and Prevention (2014) Cost of falls amongst older adults.

3. US Census Bureau (2012) Facts for features: Older Americans month.

4. Roper K (2015) South County Senior Summit: The State of Aging in Orange County. Conference sponsored by The Orange County Office on Aging and Orange County Community Services, Laguna Hills, CA, USA.

5. Centers for Disease Control and Prevention (2008) Preventing falls: How to develop community-based fall prevention programs for older adults.

6. Schepen S, Sen A, Painter JA, Murphy SL (2012) Relationship between fall-related efficacy and activity engagement in community-dwelling older adults: A meta-analytic review. American Journal of Occupational Therapy 66(2): 137-148.

7. Faes MC, Reelick MF, Banningh LW, DeGier M, Esselink RA, et al. (2010) Qualitative study on the impact of falling in frail older persons and family caregivers: Foundations for an intervention to prevent falls. Aging \& Mental Health 14(7): 834-842.

8. Huang H, Gau M, Lin W, Kernohan G (2003) Assessing risk of falling in older adults. Public Health Nursing 20(5): 399-411.
9. Makizako H, Shimada H, Doi T, Park H, Yoshida D, et al. (2013) Poor balance and low gray matter volume predict falls in older adults with mild cognitive impairment. Bio Med Central Neurology 13: 102.

10. Gillespie L, Robertson M, Gillespie W, Sherrington C, Gates S, Clemson L, Lamb S (2012). Interventions for preventing falls in older people living in the community (review). The Cochrane Collaboration 1-357.

11. Baruth M, Wilcox S, Wegley S, Buchner DM, Ory MG, et al. (2011) Changes in physical functioning in active living every day program for the active for life initiative. International Journal of Behavioral Medicine 18(3): 199-208.

12. Day L, Fildes B, Gordon I, Fitzharris M, Flamer H, et al. (2002). Randomized factorial trial of falls prevention among older people living in their own homes. BJM Publications 325(20): 1-6.

13. Sherrington C, Whitney JC, Lord SR, Herbert RD, Cumming RG, et al. (2008) Effective exercise for the prevention of falls: A systematic review and meta-analysis. Journal of the American Geriatrics Society 56(12): 2234-2243.

14. Robertson MC, Campbell AJ, Gardner MM, Devlin N (2002) Preventing injuries in older people by preventing falls: a meta-analysis of individual-level data. Journal of the American Geriatrics Society 50(5): 905-911.

15. Thiebaud RS, Funk MD, Abe T (2014) Home-based resistance training for older adults: A systematic review. Geriatric Gerontology International (4): 750-757.

16. Resnick B, Boltz M, Galik E, Pretzer Aboff I (2011) Restorative care nursing for older adults: A guide for all care settings.

17. Resnick B, Nigg C (2003) Testing a theoretical model of exercise behavior for older adults. Nursing Research 52(2): 80-88.

18. Kowitlawakul Y (2012) Discovery of changes in older adults. Singapore Nursing Journal 39(2): 10-14.

19. Taylor D, Hale L, Schluter P, Waters D L, Binns EE, et al. (2012) Effectiveness of tai chi as a community-based falls prevention intervention: A randomized-controlled trial. Journal of the American Geriatrics Society 60(5): 841-848.

20. US Department of Health \& Human Services (2015) Office of Disease Prevention and Health Promotion: Older Adults

21. Voukelatos, A., Psychol, M.A., Cumming, R.G., Lord, S.R. \& Rissel, C. (2007). A randomized, controlled trial of tai chi for the prevention of falls: The central Sydney tai chi trial. Journal of the American Geriatrics Society, 55, 1185-1191.

\section{Your next submission with Juniper Publishers will reach you the below assets}

- Quality Editorial service

- Swift Peer Review

- Reprints availability

- E-prints Service

- Manuscript Podcast for convenient understanding

- Global attainment for your research

- Manuscript accessibility in different formats

( Pdf, E-pub, Full Text, Audio)

- Unceasing customer service

Track the below URL for one-step submission https://juniperpublishers.com/online-submission.php 